Giovanna Paulina Posso-López; Mónica Cecilia Meléndez-Herrera; Silvana Paola Parra-Robalino

http://dx.doi.org/10.35381/s.v.v4i8.981

\title{
Autocuidado de los Adultos Mayores relacionados con sus características sociodemográficas
}

Self-care of Older Adults related to their sociodemographic characteristics

Giovanna Paulina Posso-López

gposso9410@uta.edu.ec

Postgrado de Medicina Familiar y Comunitaria, Facultad de Ciencias de la Salud, Universidad Técnica de Ambato, Ambato

Ecuador

https://orcid.org/0000-0003-1639-1715

Mónica Cecilia Meléndez-Herrera

monica.melendez@hgl.mspz3.gob.ec

Postgrado de Medicina Familiar y Comunitaria, Facultad de Ciencias de la Salud, Universidad Técnica de Ambato, Ambato

Ecuador

https://orcid.org/0000-0002-1728-9555

Silvana Paola Parra-Robalino

sparra699@uta.edu.ec

Postgrado de Medicina Familiar y Comunitaria, Facultad de Ciencias de la Salud, Universidad Técnica de Ambato, Ambato

Ecuador

https://orcid.org/0000-0002-1231-8812

Recepción: 15 de junio 2020

Revisado: 26 de julio 2020

Aprobación: 20 de agosto 2020

Publicación: 01 de septiembre 2020 


\section{RESUMEN}

Objetivo: determinar el nivel de autocuidado y sus características sociodemográficas relacionadas. Método: cuantitativo, descriptivo, de corte transversal de los adultos mayores de la parroquia Eloy Alfaro, Latacunga, la muestra del estudio estuvo compuesta por 73 ancianos. Resultados: resalta significativamente en el nivel de analfabetismo en un $60,27 \%$. Conclusión: el conocimiento de las condiciones sociodemográfico y de su autocuidado de estos ancianos favorece la implementación de acciones específicas, para este intervalo etario, por los profesionales de salud que trabajan en el Centro de Salud que pertenece a esta población.

Descriptores: Anciano; servicios de salud para ancianos; epidemiología. (Fuente: DeCS).

\section{ABSTRACT}

Objective: to determine the level of self-care and its related sociodemographic characteristics. Method: quantitative, descriptive, cross-sectional of the elderly of the Eloy Alfaro parish, Latacunga, the study sample was composed of 73 elderly people. Results: stands out significantly in the level of illiteracy in $60.27 \%$. Conclusion: knowledge of the sociodemographic conditions and their self-care of these elderly people favors the implementation of specific actions, for this age range, by the health professionals who work in the Health Center that belongs to this population.

Descriptores: Aged; health services for the aged; epidemiology. (Source: DeCS). 
Revista Arbitrada Interdisciplinaria de Ciencias de la Salud. SALUD Y VIDA

Volumen 4. Número 8. Año 4. Julio - Diciembre 2020

Hecho el depósito de Ley: FA2016000010

ISSN: 2610-8038

FUNDACIÓN KOINONIA (F.K).

Santa Ana de Coro, Venezuela.

Giovanna Paulina Posso-López; Mónica Cecilia Meléndez-Herrera; Silvana Paola Parra-Robalino

\section{INTRODUCCIÓN}

El fenómeno mundial del envejecimiento está tomando mayor fuerza e importancia. Debido al aumento de la población de adultos mayores, lo cual incrementa las demandas sanitarias generalmente determinados por su capacidad de dependencia. Lo cual hace que hay el interés de analizar los diferentes espacios relacionados con el envejecimiento 1.

En Ecuador la estrategia de Atención Primaria en Salud Renovada se encuentra enfatizando en una atención integral al adulto mayor por medio de estrategias que permitan producir mejoras sostenibles y equitativas ${ }^{2}$. Se proyecta que en el año 2025; en los países de Latinoamérica que el $15 \%$ de su población o más su población superará sobre los 60 años, por tal motivo esta población vulnerable debe ocupar un lugar primordial y prioritario en todo Gobierno sobre todo es sus sistemas de salud y de economía ${ }^{3}$. El envejecimiento poblacional es un fenómeno que va creciendo y tiene impacto político, social, económico y en el sector salud representando un desafío mundialista sobre todo en los países en vías de desarrollo donde la transición demográfica ha sido de manera abrupta sin dar tiempo para que se puedan reorganizar y poder implementar servicios de acorde a las necesidades de esta población ${ }^{4}$. La capacidad funcional del adulto mayor es un excelente predictor de eventos perjudiciales a un futuro cercano, con riesgo de crear dependencia, discapacidades e incluso predictor de mortalidad ${ }^{5}$. Siendo esto un problema primordial que se debe enfrentar como país, en conjunto tanto en país mundialistas y del tercer mundo; debido, a que se requiere mayor esfuerzo, reorganizar políticas de salud y de economía ${ }^{5}$.

El adulto mayor por ser un grupo vulnerable se debe garantizar la protección integral de esta población garantizando su derecho a la igualdad y respeto en todos sus ámbitos ${ }^{6}$. El autocuidado es primordial en todo momento y aún más en esta etapa última de la vida. Este concepto se refiere a toda actividad asimilada por los sujetos orientada ya a un objetivo específico ${ }^{7}$. El autocuidado se refiere a las actividades diarias que hacen los individuos, la familia y su comunidad, todo con un objetivo común que es la promoción 
de la salud, prevención de enfermedades, en caso de existir evitar que aumente y en lo posible restablecer la salud ${ }^{8}$.

Durante el proceso de envejecimiento se evidencia cambios progresivos poniendo en riesgo que exista la pérdida de autonomía, la misma que se puede ver afectada por factores físicos, ambientales, sociales económicos y psicológicos ${ }^{9}$. Por tal motivo es primordial promover el autocuidado en este grupo vulnerable con el fin de lograr una buena calidad de vida del Adulto mayor ${ }^{10}$. Actualmente es innegable el derecho que tienen los adultos mayores para poder participar en los programas de salud tanto a nivel público como privado; donde el autocuidado es el pilar fundamental para lograr y cumplir los objetivos establecidos por cada individuo o la sociedad, con el fin de lograr una población adulta mayor saludable ${ }^{11}$.

En conclusión, el autocuidado se refiere a la responsabilidad personal de cuidar la salud de forma permanente, para alcanzar una mejor calidad de vida ${ }^{7}$. La autonomía e independencia crean un autocuidado aceptable para lograr un envejecimiento exitoso. Debemos fomentar en todo adulto mayor la capacidad de cuidado y responsabilidad de su salud ya que la mayor parte de esta población tienen la capacidad mental y física necesaria para mantener su autocuidado 121314 .

El autocuidado del adulto mayor involucra en su mayor parte al adulto mayor y no sólo es obligación de los profesionales de la salud. La familia también es punto clave en el autocuidado de sus adultos mayores promoviendo actividades que implican responsabilidad sobre el cuidado de la salud ${ }^{15} 16$.

Por lo anteriormente expuesto, la presente investigación tiene como objetivo determinar el nivel de autocuidado y sus características sociodemográficas relacionadas. 
Revista Arbitrada Interdisciplinaria de Ciencias de la Salud. SALUD Y VIDA

Volumen 4. Número 8. Año 4. Julio - Diciembre 2020

Hecho el depósito de Ley: FA2016000010

ISSN: 2610-8038

FUNDACIÓN KOINONIA (F.K).

Santa Ana de Coro, Venezuela.

Giovanna Paulina Posso-López; Mónica Cecilia Meléndez-Herrera; Silvana Paola Parra-Robalino

\section{MÉTODO Y MATERIALES}

\section{Dimensión espacial de la investigación}

Se desarrolló en Latacunga, parroquia Eloy Alfaro, del Cantón Latacunga de la Provincia Cotopaxi del Ecuador, específicamente en el Centro de Salud Loma Grande.

\section{Tipo de investigación}

Es un estudio de tipo descriptivo que cuantifica y analiza las diferentes variables por medio de test validados por la OMS, (Organización Mundial de la Salud) de los Adultos mayores.

\section{Muestra poblacional}

Para la selección de la muestra se utilizó las fichas familiares donde se seleccionó a todas las personas adultas mayores, siendo esta un número poblacional de 73 personas que se encuentran con más de 65 años de edad, siendo consideradas adultas mayores.

\section{Recopilación de información}

La información fue obtenida por medio de los test, aplicados por el personal del Centro de Salud Loma Grande. El instrumento de recolección de datos fue utilizado el Test de Katz, el Test de Lawton y Brody, el Test CYPAC-AM (capacidad y percepción del autocuidado del adulto mayor) el Test de Katz y de Lawton valoran la capacidad funcional del Adulto mayor mediante las actividades básicas de la vida diaria y las instrumentales. El test de CYPAC-AM es utilizado para valorar la capacidad de percepción de autocuidado del Adulto Mayor, el mismo permite identificar si el adulto mayor tiene un autocuidado adecuado, parcialmente adecuado, percepción de autocuidado inadecuado, sin capacidad de autocuidado. 
Giovanna Paulina Posso-López; Mónica Cecilia Meléndez-Herrera; Silvana Paola Parra-Robalino

\section{Análisis estadístico}

Los datos fueron procesados descriptivamente en el sistema SPSS versión 25.

\section{Comité de ética}

Se declara que no se experimentó con humanos o animales.

\section{RESULTADOS}

\section{Características demográficas.}

\section{Tabla 1.}

Clasificación por grupos de edad.

\begin{tabular}{|l|c|c|c|}
\hline \multicolumn{1}{|c|}{$\begin{array}{c}\text { Clasificación Del Adulto } \\
\text { Mayor }\end{array}$} & Edad & Frecuencia & Porcentaje \\
\hline Adulto mayor anciano & 65 a 74 años & 25 & 34,2 \\
\hline adulto mayor maduro & $\mathbf{7 5}$ a 84 años & 35 & 47,9 \\
\hline octogenario & $\mathbf{8 5}$ a 89 años & 4 & 5,5 \\
\hline NONAGENARIO & $>90$ años & 9 & 12,13 \\
\hline
\end{tabular}

Mayoritariamente se encuentran en el rango de 75 a 84 años con un 47,9\%. 


\section{Tabla 2.}

Clasificación por sexo y por edad.

\begin{tabular}{|l|l|c|r|r|}
\hline \multicolumn{1}{|c|}{ CLASIFICACIÓN } & \multicolumn{1}{|c|}{$\begin{array}{c}\text { ADULTO MAYOR } \\
\text { ANCIANO }\end{array}$} & $\begin{array}{c}\text { ADULTO } \\
\text { MAYOR } \\
\text { MADURO }\end{array}$ & OCTOGENARIO & NONAGENARIOS \\
\hline SEXO FEMENINO & $23,3 \%(17)$ & $32,9 \%(24)$ & $4,1 \%(3)$ & $8,2 \%(6)$ \\
\hline SEXO MASCULINO & $11 \%(8)$ & $15,1 \%(11)$ & $1,4 \%(1)$ & $4,1 \%(3)$ \\
\hline TOTAL & $34,2 \%(25)$ & $47,9 \%(35)$ & $4(5,5 \%)$ & $12,3 \%(9)$ \\
\hline
\end{tabular}

Resalta el sexo femenino y adulto mayor con $32,9 \%$ y $15,1 \%$

\section{Tabla 3.}

Estado civil de los Adultos Mayores.

\begin{tabular}{lll}
\hline Estado civil & $\mathbf{N}$ & $\%$ \\
\hline Soltero & 3 & 4,11 \\
Casado & 55 & 75,34 \\
Unido & 0 & 0,00 \\
Separado & 0 & 0,00 \\
Viudo & 15 & 20,55 \\
\hline Total & $\mathbf{7 3}$ & $\mathbf{1 0 0 , 0 0}$ \\
\hline
\end{tabular}

El adulto mayor casado predomina en este indicador. 


\section{Tabla 4.}

El nivel de educación en el sector es muy bajo, sobresale el analfabetismo.

\begin{tabular}{|l|r|r|}
\hline Nivel educativo & $\mathbf{N}$ & $\%$ \\
\hline Analfabeto & 44 & 60,27 \\
\hline Educación Básica & 27 & 36,99 \\
\hline $\begin{array}{l}\text { Secundaria } \\
\text { incompleta }\end{array}$ & 2 & 2,74 \\
\hline Bachillerato & 0 & 0,00 \\
\hline Superior & 0 & 0,00 \\
\hline Total & $\mathbf{7 3}$ & $\mathbf{1 0 0 , 0 0}$ \\
\hline
\end{tabular}

Siendo significativamente en el nivel de analfabetismo en un 60,27\%.

\section{Tabla 5.}

Ocupación de los adultos mayores de San Juan, Latacunga.

\begin{tabular}{lll}
\hline Ocupación & $\mathbf{n}$ & $\%$ \\
\hline Agricultor & 30 & 41,10 \\
Quehaceres & & \\
domésticos & 30 & 41,10 \\
Ninguna & 10 & 13,70 \\
Otros & 3 & 4,11 \\
\hline Total & $\mathbf{7 3}$ & $\mathbf{1 0 0 , 0 0}$ \\
\hline
\end{tabular}

Se deduce que las mujeres realizan quehaceres domésticos y los hombres se dedican a la agricultura. 


\section{Tabla 6.}

Autopercepción del grado de Autocuidado según el Test de CYPAC AM en los adultos mayores de San Juan, Latacunga.

\begin{tabular}{lrr}
\hline Autocuidado & N & \multicolumn{2}{c}{$\%$} \\
\hline Adecuado & 22 & 30,14 \\
Aceptable & 31 & 42,47 \\
Inadecuado & 8 & 10,96 \\
Incapacidad de cuidado & 12 & 16,44 \\
\hline Total & $\mathbf{7 3}$ & $\mathbf{1 0 0 , 0 0}$ \\
\hline
\end{tabular}

Se visualiza mayor proporción en aceptable con $42,47 \%$ y adecuado con $30,14 \%$.

\section{DISCUSIÓN}

En Ecuador la población femenina (53,03\%) es superior a la masculina $(46,97 \%)$ en el grupo etario de adultos mayores, evidentemente con una diferencia menor, puesto que, en un estudio con un universo menor, la proporción entre variables tiende a ser mayor, dando como resultado una mayor desviación estándar y mayor es la dispersión de la población. Pero los datos observados de igual manera nos indica que existe predominio en el grupo de estudio del sexo femenino.

Al respecto nace en la actualidad el concepto de "feminización del envejecimiento", fenómeno que responde a la mayor cantidad de mujeres respecto a los hombres en casi todos los países a nivel mundial.

Además, 1 de cada 4 personas adultas mayores es analfabeta. Esta situación es mayor en las mujeres, cuyo porcentaje llega al $29 \%$, y en el caso de los hombres, es del $20 \%$. En comparación con los datos obtenidos en nuestro estudio podemos observar que existe un elevado porcentaje que supera el $50 \%$ considerados analfabetos y un porcentaje que 
Revista Arbitrada Interdisciplinaria de Ciencias de la Salud. SALUD Y VIDA

Volumen 4. Número 8. Año 4. Julio - Diciembre 2020

Hecho el depósito de Ley: FA2016000010

ISSN: 2610-8038

FUNDACIÓN KOINONIA (F.K).

Santa Ana de Coro, Venezuela.

Giovanna Paulina Posso-López; Mónica Cecilia Meléndez-Herrera; Silvana Paola Parra-Robalino

no supera el $2 \%$ ha cursado la secundaria, pero no la ha completado. Las cifras en comparación a otros estudios se dispersan debido a que la muestra en investigación es pequeña. En América Latina la religión tiene gran tendencia en las culturas de predominio es la católica con el $87,20 \%$ de creyentes. Al igual que en este estudio, se demuestra una tendencia similar.

\section{FINANCIAMIENTO}

Autofinanciado

\section{AGRADECIMIENTOS}

A Centro de Salud Loma Grande; por su apoyo en la realización de la investigación.

\section{REFERENCIAS}

1. Can Valle AR, Sarabia Alcocer B, Guerrero Ceh JG. Autocuidado en las personas mayores de la Ciudad de San Francisco de Campeche / Self-care in older persons of San Francisco City of Campeche. RIDE [Internet]. 2016, 6(11):721 -739.

2. Dirección Nacional de Articulación y Manejo del Sistema Nacional de Salud y de la Red Pública. Modelo de atención integral del sistema nacional de salud. Primera edición. Ecuador. Ministerio de Salud Pública. (2012).

3. Aranibar, P., De Chile, S., Latinoamericano, C. \& De Demografía, C. Acercamiento conceptual a la situación del adulto mayor en América Latina población y desarrollo Proyecto Regional de Población CELADE-FNUAP. (Fondo de Población de las Naciones Unidas). (2001).

4. Loredo-Figueroa M.T., Gallegos-Torres R.M., Xeque-Morales A.S., Palomé-Vega G., Juárez-Lira A. Nivel de dependencia, autocuidado y calidad de vida del adulto mayor. Enferm. Univ. [revista en la Internet]. 2016; 13(3): 159-165.

5. Paredes-Arturo Yenny Vicky, Yarce-Pinzón Eunice, Aguirre-Acevedo Daniel Camilo. Funcionalidad y factores asociados en el adulto mayor de la ciudad San Juan de Pasto, Colombia. Rev. Cienc. Salud . [Internet]. 2018; 16(1): 114-128. 
Revista Arbitrada Interdisciplinaria de Ciencias de la Salud. SALUD Y VIDA

Volumen 4. Número 8. Año 4. Julio - Diciembre 2020

Hecho el depósito de Ley: FA2016000010

ISSN: 2610-8038

FUNDACIÓN KOINONIA (F.K).

Santa Ana de Coro, Venezuela.

Giovanna Paulina Posso-López; Mónica Cecilia Meléndez-Herrera; Silvana Paola Parra-Robalino

6. Leite Marinês Tambara, Castioni Daniani, Kirchner Rosane Maria, Hildebrandt Leila Mariza. Capacidad funcional y nivel cognitivo de adultos mayores residentes en una comunidad en el sur de Brasil. Enferm. glob. [Internet]. 2015; 14(37): 111.

7. Troncoso-Pantoja Claudia, Muñoz-Roa Mirna, Amaya-Placencia Juan Pablo, DíazAedo Fredy, Sotomayor-Castro Mauricio, Jerez-Salinas Alejandra. Mejorando la calidad de vida en adultos mayores a través de la experiencia universitaria. Gerokomos. [Internet]. 2019; 30(3): 113-118.

8. Sabando Mera VM, Zambrano Vera MF, Molina Sabando L, Loor Vega MP. Promoción de estilos de vida saludables en la investigación e intervención de la salud familiar intercultural en el adulto mayor. YACHANA. [Internet]. 2018; 5(3): 23-32.

9. Villafuerte Reinante Janet, Alonso Abatt Yenny, Alonso Vila Yisel, Alcaide Guardado Yuleydi, Leyva Betancourt Isis, Arteaga Cuéllar Yoleisy. El bienestar y calidad de vida del adulto mayor, un reto para la acción intersectorial. Medisur. [Internet]. 2017; 15(1): 85-92.

10. Vargas Santillán Ma de Lourdes, Arana Gómez Beatriz, Garcia Hernández Maria de Lourdes, Ruelas González Guadalupe, Melguizo Herrera Estela, Ruiz Martínez Ana Olivia. Prácticas de autocuidado en adultos mayores: un estudio cualitativo en una población mexicana. Rev. Enf. Ref. . [Internet]. 2018; serlV(16): 117-126.

11. Vera López, Juana Isabel. Situación actual de los Derechos humanos en la normativa del adulto mayor en América Latina y México. Nómadas. Critical Journal of Social and Juridical Sciences [Internet]. 2016;47(1).

12. Laguado Jaimes Elveny, Camargo Hernández Katherine del Consuelo, Campo Torregroza Etilvia, Martín Carbonell Marta de la Caridad. Funcionalidad y grado de dependencia en los adultos mayores institucionalizados en centros de bienestar. Gerokomos . [Internet]. 2017; 28(3): 135-141.

13. Millán Méndez Israel E. Evaluación de la capacidad y percepción de autocuidado del adulto mayor en la comunidad. Rev Cubana Enfermer. [Internet]. 2010; 26(4): 202-234.

14. Chiriboga Efrén Viteri, Terranova Barrezueta Aida Elizabeth, Velis Aguirre Lazara Milagros. Funcionalidad familiar y autoestima del adulto mayor, en situación de maltrato. Un estudio participativo comunitario. Rev. Lasallista Investig. [Internet]. 2018; 15(2): 300-314. 
Revista Arbitrada Interdisciplinaria de Ciencias de la Salud. SALUD Y VIDA

Volumen 4. Número 8. Año 4. Julio - Diciembre 2020

Hecho el depósito de Ley: FA2016000010

ISSN: 2610-8038

FUNDACIÓN KOINONIA (F.K).

Santa Ana de Coro, Venezuela.

Giovanna Paulina Posso-López; Mónica Cecilia Meléndez-Herrera; Silvana Paola Parra-Robalino

15. Aliaga-Díaz E, Cuba-Fuentes S, Mar-Meza M. Promoción de la salud y prevención de las enfermedades para un envejecimiento activo y con calidad de vida. [Health promotion and disease prevention for active aging that preserves quality of life]. Rev Peru Med Exp Salud Publica. 2016; 33(2):311-20.

16. Rivera-Ledesma A, Montero-Lòpez Lena M. Ajuste psicológico y vida religiosa en Adultos Mayores. Univ Psychol. [Internet]. 2014;13(3).

(C2020 por los autores. Este artículo es de acceso abierto y distribuido según los términos y condiciones de la licencia Creative Commons Atribución-NoComercial-Compartirlgual 4.0 Internacional (CC BY-NC-SA 4.0)

(https://creativecommons.org/licenses/by-nc-sa/4.0/). 